\title{
Parametric Reduction for Simplified Posture Classification Model
}

\author{
Peter Dabnichki*. Arnold Baca**, Martin Groeber***, Maria Del Pilar Garcia Souto**** \\ *School of Engineering, RMIT University, Melbourne, 3001, Australia \\ (Tel: +61399257278; e-mail: peter.dabnichki@rmit.edu.au) \\ ** Dept of Biomechanics and Computer Science in Sport, University of Vienna \\ A-1150Vienna, Austria (arnold.baca@univie.ac.at) \\ *** Dept of Biomechanics and Computer Science in Sport, University of Vienna \\ A-1150Vienna, Austria (martin.groeber@univie.ac.at) \\ ****Dept of Medical Physics and Biomedical Engineering, University College London \\ London,WC1E6BT, UK, (e-mail: p.garciasouto@ucl.ac.uk)
}

\section{INTRODUCTION}

The continuous increase in sedentary work over the last decades has resulted in a higher risk of hypertension, overweight, obesity, diabetes and musculoskeletal problems. Furthermore prolonged seating affects the thermal comfort requirements commonly leading to unhealthy workplace temperatures and conditions (Garcia Souto et al, 2013; Michael et al, 2017).

Traditionally sitting related research has been an area of interest for patients with impaired mobility (Dabnichki \& Taktak, 1998). More recently a variety of interventions have been proposed and developed ranging from activity programs aiming to reduce sedentary time to posture recognition tools prompting the user to avoid ergonomically inappropriate postures (Schwartz et al. 2013). To date most posture recognition models have predominantly relied on video based approaches that are controversial for implementation during work and travel..

These models play an essential role in bridging the gap between movement/posture data and static and dynamic analysis of forces occurring. However, all these models require different number of inputs and are not easily applied in practical situations (Dabnichki et al, 1998).

In this work we demonstrate how through model sensitivity analysis we reduce the required inputs, the sampling frequency and inherent complexity of the anthropometric model to develop a simple method of posture recognition. We do not discuss the developed classification that is compliant with ergonomic practice as it is outside the scope of this work.

\section{MODEL BUILDING AND COMPLEXITY REDUCTION}

The model was built through the use of biomechanical analysis to observe and classify postures using an imaged based system with integrated GRF data collection. The purpose of this process was to reduce the complexity of the model and ultimately eliminate the use of image data. We describe the steps in this process below.

\subsection{Combined image and force data collection and analysis}

In order to gain better insight into the kinetic trace of the postures considered we mounted an office chair of a force plate and conducted integrated data collection with on-line force analysis.

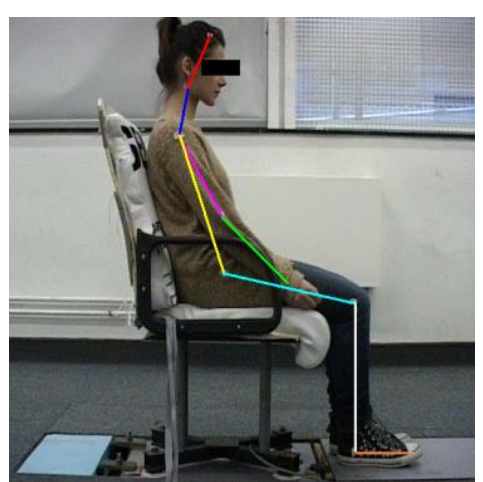

Fig.1. Data collection set-up for automated posture analysis.

which in the simplified model form is

$$
\overrightarrow{G R F} \times \overrightarrow{C O P}=\overrightarrow{w_{1}} \times \overrightarrow{C O M_{1}}+\overrightarrow{w_{2}} \times \overrightarrow{C O M_{2}}+\overrightarrow{w_{3}} \times \overrightarrow{C O M_{3}}+\vec{f} \times \overrightarrow{d_{f}}=0
$$

where we use the standard notation for the ground reaction force, centre of mass and centre of pressure. The $w_{i}$ indicates the respective weights of the 3 modified segments and $f$ is the generalkised friction force and $d_{f}$ is the distance of its point of application from the origin of the reference system

\subsection{Detailed force analysis}

In the second stage of the process we looked into the influence of the position and movement of different parts on the body on the balace of forces and moments (sensitivity analysis) produced some surprising outcomes for us and luckily they were very pleasing. 


\subsection{Analysis of body balance}

Having achieved model reduction we needed to find out whther a posture described this way complies with the two fundamental mechanical principles. i.e. the balance of forces and the balance of moments. Hence it was essential to establish where the imaginary centre of mass should be pllaced. Here we need to point out that the analysis of this assumes $3 \mathrm{D}$ coordinates as the medial-lateral movement is considred as adaptive parameter.

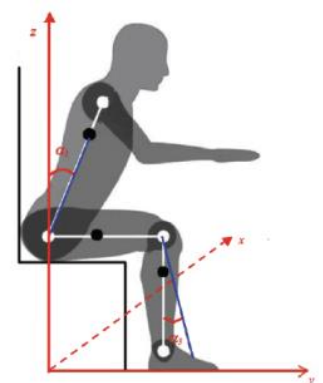

Fig.2. Simplified anthropometric model.

of the centre of mass of the Upper Body Segment (with a small perturbation parameter). Similarly the location of the centre of mass of the Lower Body was assumed to coincide with the location of the centre of mass of the Calves as their mass is an order higher than that of the feet, although there is a slight adjustment made in the sagittal plane.

This reduction means that to build a model for an individual, one needs just height and weight. Accuracy would improve if gender is indicated but it is not a firm requirement.

Furthermore depending on the implementation there is a built-in procedure for weight verification so strictly speaking weight input is also optional.

\section{MODEL OVERVIEW AND IMPLEMENTATION}

The model described in the previous was tested in an on-line automated system in several stages. The first stage was to test it on random samples of the pre-collected data and compare to visual classification already tagged. Again using the existing data an "inverse" simulation was then run to obtain the CoP position based on the visual input from the modified body segments. The reliability criterion was the position of the centre of pressure and whether it is identifiable with the assumed posture.

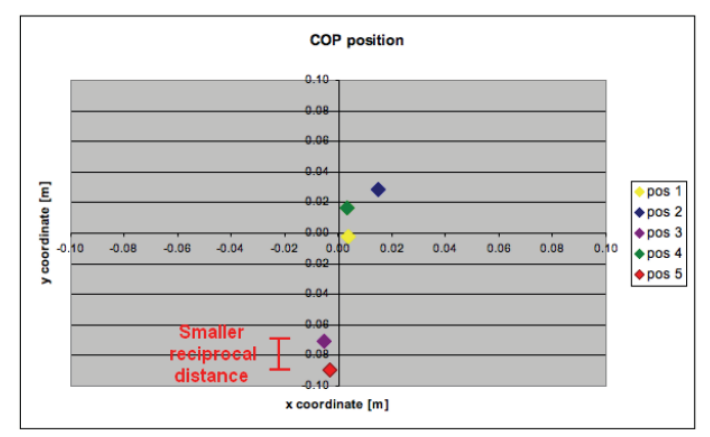

Fig. 3. Position of the centre of pressure on the force plate.
The results yielded for the posture classification proved to be clear and allow unambiguous classification in about $85 \%$ of the cases. As already discussed the dominant effect is in the sagittal plane and it is used as the fastest indicator. The

\section{SYSTEM CONSTRAINTS AND FURTHER DEVELOPMENT}

The results that the current work produced are pleasing as with a small degree of personalisation the accuracy exceeds $90 \%$. However, the algorithm heavily relies on the accuracy of the force data measurement of all 12 force components (and this number cannot be reduced without compromising the COP estimate which in turn will drastically reduce the success rate in posture recognition. From the very beginning we have been well aware that the use of a force plate in everyday environment is neither practical nor likely.

The reduced reliability is likely to prompt us to use more elaborate artificial intelligence based methods combined with system self-calibration. The system is likely to only use the model in the initial assessment and then use a learning algorithm to adapt to the individual behaviour. Still the relatively low frequency of action provides good analytical opportunities to reduce the guessing element in the process of classification. We are also looked into possibility of a chair mounted force plate equivalent to measure the total force.

On the theoretical front we are looking into the topological characterisation of the areas of uncertainty for the key points allocation to reduce the grey areas of uncertain classification. This is particularly important when a new subject is introduced.

\section{REFERENCES}

Dabnichki, P. and Taktak D. (1998) Pressure variation under the ischial tuberosity during a push cycle, Medical Enineering \& Physics, 20(4), 242-56.

Dabnichki, P., Lauder, M., Aritan, S., 1997 Accuracy evaluation of an on-line kinematic system via dynamic tests, Journal of Medical Engineering \& Technology, 21(2), 53-66,

Garcia-Souto, M. and Dabnichki, P., 2016, Core and local skin temperature: 3-24 months old toddlers and comparison to adults. Building and Environment, 104, 286-295

Garcia-Souto M. and Dabnichki P. (2013) Skin temperature distribution and thermoregulatory response during prolonged seating. Building and Environment, 69, 14-21

Michael K, Garcia-Souto MDP and Dabnichki, P. (2017) An investigation of the suitability of Artificial Neural Networks for the prediction of core and local skin temperatures when trained with a large and genderbalanced database. Appl Soft Comp, 50, 327-343

Schwartz, B. (2015) Cognitive and biomechanical effects of postural changes in office environments. Proceeding of the 61th Spring-Congress on the Society for Ergonomics and Work Science, Karlsruhe, Germany 